Research Article

\title{
Characterization of Elastic and Plastic Behaviors in Steel Plate Based on Eddy Current Technique Using a Portable Impedance Analyzer
}

\author{
Meng Fanlin, Liu Xiucheng, Wang Heyun, He Cunfu, and Wu Bin \\ College of Mechanical Engineering and Applied Electronics Technology, Beijing University of Technology, Beijing, China \\ Correspondence should be addressed to Liu Xiucheng; xiuchliu@bjut.edu.cn
}

Received 9 October 2017; Accepted 30 November 2017; Published 31 December 2017

Academic Editor: Andrea Cusano

Copyright (C) 2017 Meng Fanlin et al. This is an open access article distributed under the Creative Commons Attribution License, which permits unrestricted use, distribution, and reproduction in any medium, provided the original work is properly cited.

\begin{abstract}
A portable impedance analyzer (PIA) was developed based on a TiePie-HS3 device to provide the comparable impedance measurement accuracy of the Agilent 4294a impedance analyzer in the frequency range of $0 \sim 250 \mathrm{kHz}$. Then the PIA was applied to monitor the tensile stress-induced variation of the eddy current sensor's impedance in a medium-carbon steel sample. A model of equivalent magnetic field induced by the elastic stress and the number of pinning sites indicated that the inductance of the eddy current loop firstly increased with the increase in the tensile stress and then decreased at the yield point of the material. The experimental results testified that the variation of impedance amplitude, the variation of phase angle, and the shift of two featured frequencies demonstrated opposite variation trends before and after the yield point, as predicated by the model. A new parameter, which combined the impedance variation information of the selected two frequencies, was found to exhibit nearly monotonous dependency on the tensile stress in elastic and plastic stages. The new parameter together with the developed portable impedance analyzer provided the solution to identify the elastic and plastic behaviors in ferromagnetic materials in practical applications with an eddy current technique.
\end{abstract}

\section{Introduction}

Eddy current techniques are typically applied for nondestructive evaluation of metals $[1,2]$. It is well known that microstructures and their stress states determine the electrical conductivity and magnetic permeability of ferromagnetic materials $[3,4]$. Eddy current techniques have the potential to evaluate the microstructure change [5-7], state of applied/residual stress $[8,9]$, and plasticity $[10,11]$ in ferromagnetic materials. The above objective parameters can be quantified based on eddy current sensor's impedance which is measured by advanced impedance analyzers such as Agilent 4294a or integrated experimental system [12]. Considering practical applications, it is necessary to develop portable impedance analyzer (PIA) with comparable accuracy and ease in operation [13].

To investigate the effects of elastic and plastic strains on eddy current response in metals by experimental tools, uniaxial tensile loading is commonly applied in the tensioned samples and the impedance variation of the eddy current sensor (ECS), which is placed near the sample surface, is recorded during the entire tension process [14]. A characterization of elastic behavior by ECS response in nonferromagnetic metals such as aluminum alloys and austenitic stainless steel was widely studied and discussed $[15,16]$. The piezoresistive effect is considered as the main cause of the variation of eddy current response. In plastic deformation stage, both the additional microstructure change and the stress influence the ECS response. As a result, some features of ECS response, which are uncorrelated to elastic strain, become sensitive to plastic strain [17].

Similarly, in ferromagnetic materials, the influences of dominate magnetoelastic effect and plastic deformation on the ECS impedance shift were investigated. Schoenekess et al. [18] pointed out that under the small magnetic field strength, the reactance of ECS impedance is more sensitive to the elastic stress than the resistance. Dahia et al. [19] alternatively measured the magnetic permeability of the 
ferromagnetic iron-cobalt alloy under tensile elastic stress. Both the permeability and the ECS impedance amplitude were found to monotonously increase with the increase in the elastic stress. On the contrary, Cai et al. [20] reported that the increasing residual plastic strain decreased the permeability of carbon steels. The observed monotonous dependency of eddy current signal features on individual elastic stress or plastic strain may allow nondestructive evaluations. In order to find methods for identifying the deformation stage based on ECS response and separating the contributions of elastic and plastic strains to ECS responses, it is necessary to explore the eddy impedance during the entire tension process including both the elastic and plastic stages [21].

In the study, the effects of elastic and plastic deformations in medium carbon steel on the impedance shift of an eddy current sensor were experimentally investigated. Firstly, a portable impedance analyzer based on TiePie-HS3 device was developed. Performance test results indicate that in the concerned frequency range, the developed PIA possessed comparable measurement accuracy with the commercial Agilent 4294a impedance analyzer. Secondly, several featured impedance parameters were found to be sensitive to the applied tensile stress, and completely opposite conclusions about the dependency of featured impedance parameters on the tensile stress were obtained from the plastic stage against that of the elastic stage. Finally, in order to identify the elastic or plastic stage in the steel, the new parameter, which combines the impedance variation information of the two frequencies, is introduced to roughly figure out the deformation types.

\section{Elastic and Plastic Characterizations Based on the Eddy Current Method}

The piezoresistive effect describes the change in the electrical resistance of a material caused by the applied stress, whereas the magnetoelastic effect refers to the change in magnetization and consequent inductance of ferromagnetic materials caused by the applied stress. Eddy current transformer model is usually employed to illustrate the relationships between the ECS impedance and the parameters (resistance, inductance, etc.) of the induced eddy current loop in the tested ferromagnetic materials. According to the equations reported by García-Martín et al. [22], the resistance $\left(R_{\mathrm{e}}\right)$ and inductance $\left(L_{\mathrm{e}}\right)$ of ferromagnetic materials have complex influences on both the real $\left(R_{\mathrm{cn}}\right)$ and imaginary $\left(X_{\mathrm{cn}}\right)$ parts of the impedance of a search coil,

$$
\begin{aligned}
R_{\mathrm{cn}} & =\frac{k^{2} \omega L_{\mathrm{e}} R_{\mathrm{e}}}{R_{\mathrm{e}}^{2}+\left(L_{\mathrm{e}} \omega+I_{\mathrm{m}}\right)^{2}}, \\
X_{\mathrm{cn}} & =1-\frac{k^{2} \omega L_{\mathrm{e}}\left(L_{\mathrm{e}} \omega+I_{\mathrm{m}}\right)}{R_{\mathrm{e}}{ }^{2}+\left(L_{\mathrm{e}} \omega+I_{\mathrm{m}}\right)^{2}}
\end{aligned}
$$

where $I_{\mathrm{m}}$ and $\omega$ represent the leakage inductance of the circuit and the angular frequency, respectively. The coupling coefficient $k$ is related to the distance between the ECS and the tested sample. Hence, if an ECS is mounted onto the tested ferromagnetic sample surface without lift-off fluctuation, the shift of the ECS impedance is mainly induced by the changes in the resistance and inductance of the ferromagnetic material, which may indicate the stress or microstructure changes of the material.

The influencing mechanism of stress and microstructures on the resistance and inductance of ferromagnetic material cannot be yet revealed by analytical models. In order to find the elastic coefficients $(\eta)$ of the electrical resistance along the application direction of the elastic stress $(\sigma)$, the linear assumption is expressed as follows [23]:

$$
R_{\mathrm{e}}=R_{\mathrm{e} \mid \sigma=0}+\eta \sigma
$$

When a ferromagnetic material undergoes a plastic deformation in an applied magnetic field, the magnetization $(M)$ is induced by the equivalent field due to the applied magnetic field $\left(H_{\sigma=0}\right)$, elastic stress $\left(H_{\sigma}^{E}\right)$, and plastic deformation $\left(H_{\sigma}^{P}\right)$, respectively. Consequently, for a given dynamic magnetic field, the inductance $\left(L_{\mathrm{e}}\right)$ of eddy current loop in ferromagnetic materials is determined by the combined effect of the abovementioned equivalent fields,

$$
L_{\mathrm{e}} \propto \mu_{\text {total }}=\frac{B_{\text {total }}}{H_{\sigma=0}+H_{\sigma}^{E}+H_{\sigma}^{P}},
$$

where $\mu_{\text {total }}$ and $B_{\text {total }}$ are, respectively, the total equivalent permeability and the magnetic flux density. When the direction of the applied magnetic field is consistent with that of the uniaxial stress in the materials, the components of the equivalent field induced by the elastic stress can be expressed as follows [24]:

$$
H_{\sigma}^{E}=\frac{3}{2} \frac{\sigma}{\mu_{0}}\left(\frac{\mathrm{d} \lambda}{\mathrm{d} M}\right)_{\sigma},
$$

where $\mu_{0}$ stands for the permeability of air; the magnetostrictive coefficient $\lambda$ represents the magnetomechanical sensitivity; $(\mathrm{d} \lambda / \mathrm{d} M)_{\sigma}$ expresses the magnetostriction variation with magnetization under a constant stress. Experimental results showed that in carbon steels, the value of $(\mathrm{d} \lambda / \mathrm{d} M)_{\sigma}$ in low magnetic field slightly varies with the applied stress [25]. Therefore, the effect of this term on $H_{\sigma}^{E}$ is limited compared with the elastic stress $(\sigma)$.

According to the empirical formula, the increasing number of pinning sites, $N$, produced by the plastic deformation process is expressed as follows [25]:

$$
N=K \sigma^{4}
$$

where $K$ is a material-dependent constant. According to the previously reported model [26], the equivalent field caused by the plastic deformation can be given by

$$
H_{\sigma}^{P}=-\left(\frac{k\left\langle\varepsilon_{\pi}\right\rangle}{2 m \mu_{0}}\right) \sigma^{4},
$$

where $m$ is the magnetic moment of a domain; $\left\langle\varepsilon_{\pi}\right\rangle$ denotes the pinning energy of the site for $180^{\circ}$ domain walls. As indicated in (4) and (6), the stress in the elastic or plastic stage has the completely opposite effect on the stressinduced equivalent magnetic field. Due to the above 


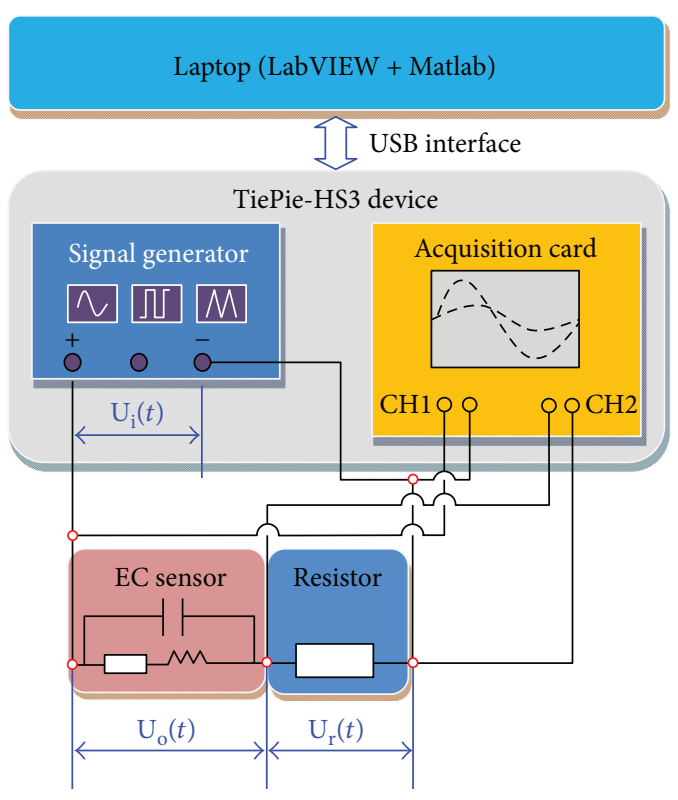

(a)

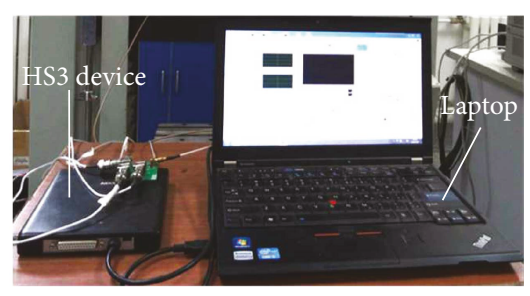

(b)

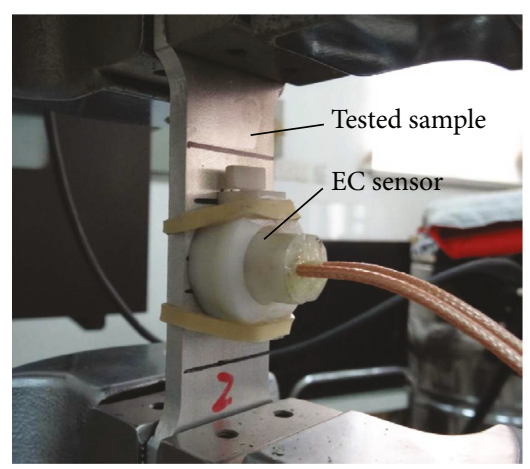

(c)

FIGURE 1: Impedance measurement circuit (a) and the portable impedance analyzer (b) designed for the eddy current sensor (c).

mechanism of the opposite effect, the relative differential permeability of carbon steels initially increases with the increase in the stress and then decreased at the yield point of the material [25].

Equations (1), (2), (3), (4), (5), and (6) link the ECS impedance with applied stress for understanding the operation principle of eddy current-based elastic and plastic deformation evaluation method. However, (1), (2), (3), (4), (5), and (6) cannot be applied in the precise prediction of stress-induced ECS impedance shift because it is difficult to determine the above parameters, such as $k,\left\langle\varepsilon_{\pi}\right\rangle$, and $m$. Furthermore, many factors such as the senor configuration, microstructure of the tested sample, and the performance of the impedance analyzer are ignored in the above model. Therefore, in most cases, the experimental calibration method was employed to investigate the effects of elastic and plastic deformations on the impedance of the ECS.

\section{Portable Impedance Analyzer}

Commercial impedance analyzers such as Agilent 4294a can simultaneously measure two independent impedance parameters of eddy current sensors, such as the real $\left(R_{\mathrm{cn}}\right)$ and imaginary $\left(X_{\mathrm{cn}}\right)$ parts of the impedance. The absolute impedance $(Z)$ and phase angle $(\theta)$ can be calculated according to the impedance vector of the equivalent series circuit, $\mathbf{Z}=R_{\mathrm{cn}}+\mathrm{j} X_{\mathrm{cn}}$. Since the commercial impedance analyzer is not convenient in practical measurements, compact impedance measurement circuits inspired by the small current measurement circuit proposed by Peairs et al. [27] were developed especially for piezoelectric elements in impedance-based SHM systems [28].

A simple and portable system was proposed to measure ECS impedance based on digital spectrum analysis method [29]. The measurement circuit is shown in Figure 1(a), and the system is composed of a TiePie-HS3 device and a laptop equipped with LabVIEW software (Figure 1(b)). The TiePie-HS3 device is comprised of a programmable signal generator and a two-channel acquisition card which communicates with the laptop and LabVIEW program through the USB interface.

A resistor with a resistance of $R$ is serially connected to the eddy current sensor. When the resistor together with the ECS is driven by a sinusoidal signal $\mathbf{U}_{\mathrm{i}}(t)$, the alternative current, $\mathbf{I}(t)$, in the circuit can be calculated as follows:

$$
\mathbf{I}(t)=\frac{\mathbf{U}_{\mathrm{r}}(t)}{R},
$$

where $\mathbf{U}_{\mathrm{r}}(t)$ represents the measured voltage of the resistor. The signals of $\mathbf{U}_{\mathbf{i}}(t)$ and $\mathbf{U}_{\mathrm{r}}(t)$ are synchronously acquired by the acquisition card. The voltage applied to the ECS, $\mathbf{U}_{\mathrm{o}}(t)$, can be expressed with the unknown impedance of the ECS, $\mathbf{Z}$, and the current, $\mathbf{I}(t)$,

$$
\begin{aligned}
\mathbf{U}_{\mathrm{o}}(t) & =\mathbf{Z} \cdot \mathbf{I}(t) \\
& =\mathbf{Z} \cdot \frac{\mathbf{U}_{\mathrm{r}}(t)}{R} .
\end{aligned}
$$

According to the Kirchoff's law, we can obtain

$$
\mathbf{U}_{\mathrm{i}}(t)-\mathbf{Z} \cdot \frac{\mathbf{U}_{\mathrm{r}}(t)}{R}-\mathbf{U}_{\mathrm{r}}(t)=0 .
$$

Thus, the impedance of the ECS is

$$
\mathbf{Z}=\left[\frac{\mathbf{U}_{\mathrm{i}}(t)}{\mathbf{U}_{\mathrm{r}}(t)}-1\right] R
$$




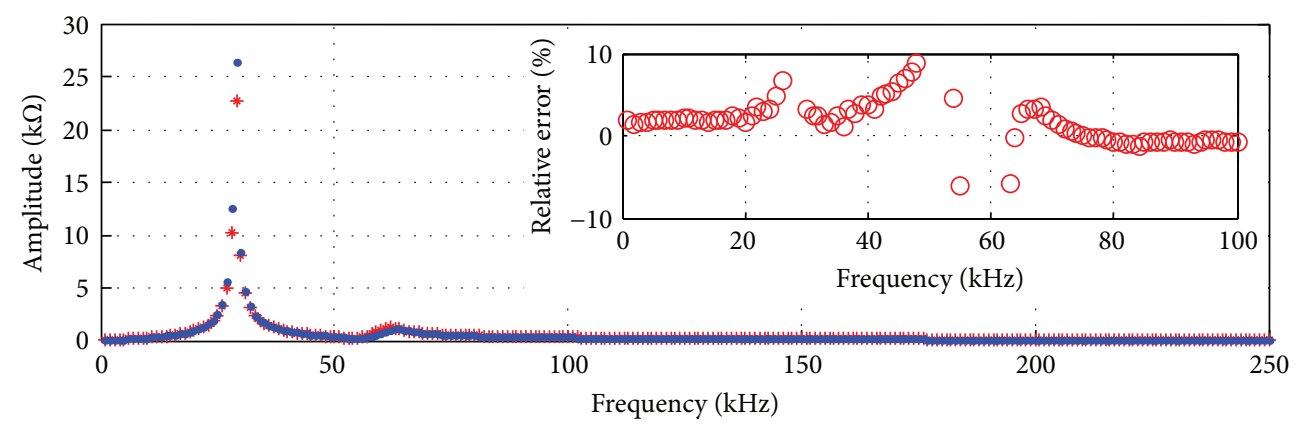

* Agilent 4294a

- PIA

(a)

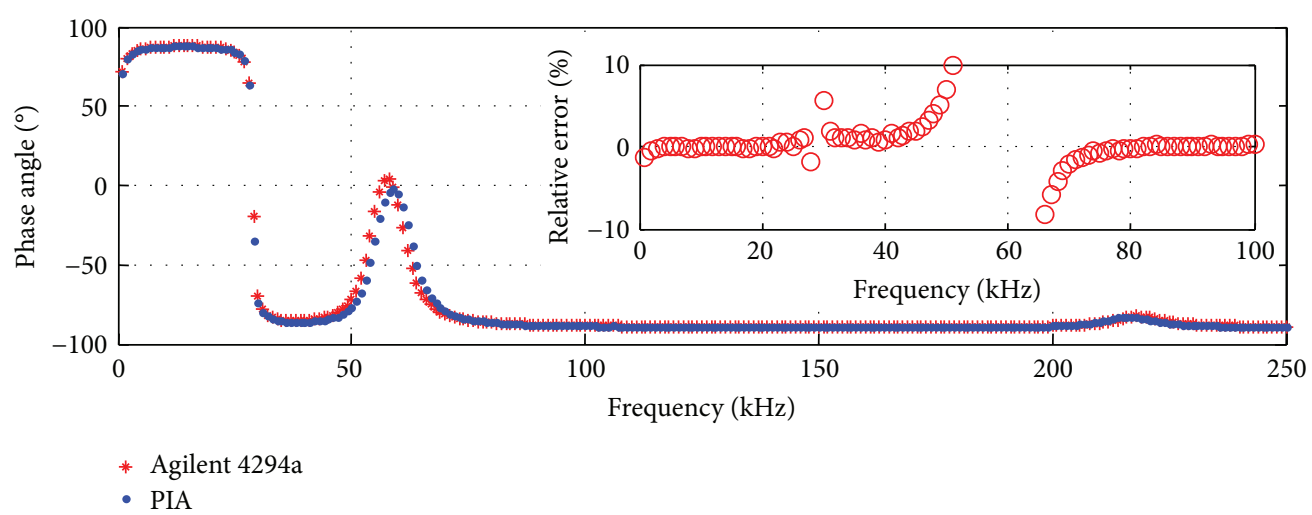

(b)

Figure 2: Comparison of impedance amplitude (a) and phase angle (b) measured by Agilent 4294a and the developed portable impedance analyzer.

The digital spectrum analysis technique was used to analyze the signals of $\mathbf{U}_{\mathrm{i}}(t)$ and $\mathbf{U}_{\mathrm{r}}(t)$ in the MATLAB environment in the LabVIEW program. The amplitude ratio and phase difference between the signal of $\mathbf{U}_{\mathrm{i}}(t)$ and $\mathbf{U}_{\mathrm{r}}(t)$ at the operating frequency were extracted and then substituted into (10) to calculate the value of ECS impedance.

The impedance measurement accuracy of the device is determined by the average measurement number and especially the value of the current-sampling resistor. The previously reported results [29] showed that the measured voltage signal was seriously disturbed by electrical noises if the value of the resistance was less than $20 \Omega$. When the resistance of $R$ was larger than $300 \Omega$, both the measured amplitude and phase angle were much higher than the values measured by the Agilent 4294a impedance analyzer due to the nonignorable current shunt effect of the inner resistor of the acquisition card. A relative error of $\pm 10 \%$ between the results measured by the developed portable and Agilent 4294a impedance analyzer could be achieved when the resistance of $R$ was in the range of $20 \sim 300 \Omega$. The increase in average measurement time could enhance the measurement accuracy at the cost of longer acquisition time. Root-meansquare deviation (RMSD) among the impedance curves measured in different average measurement time was estimated. An uncertainty of $\pm 0.25 \%$ for the impedance measurement was reported when the average time was selected to be three.
The performance comparison between the developed portable impedance analyzer (PIA) and the Agilent 4294a impedance analyzer in ECS impedance measurement is shown in Figure 2. The ECS is composed of a U-shape ferrite core whose legs are wounded with copper coils. The diameter of the coil is around $0.13 \mathrm{~mm}$, and the total number of the coil turns is about 800 . In the test, the average time is fixed as three and the resistance of the current sampling resistor is selected as $50 \Omega$. Minor deviations between the measured resonant frequencies are attributed to the differences in the electrical measurement circuit. At most of the operating frequencies beyond the resonant frequency range, the relative errors of the amplitude and phase angle measured by the PIA are small compared to those measured by the Agilent $4294 \mathrm{a}$ impedance analyzer. For instance, in the ranges of $0 \sim 20 \mathrm{kHz}$ and $80 \sim 100 \mathrm{kHz}$, the estimated relative errors of amplitude and phase angle are, respectively, less than $2.3 \%$ and $1.3 \%$ (the inserts in Figures 2(a) and 2(b)). The results prove that the developed PIA can provide very high impedance measurement accuracy for eddy current testing.

\section{Experiments and Discussion}

Medium carbon steel comprising, by weight, $0.48 \%$ of $\mathrm{C}$, $0.18 \%$ of $\mathrm{Si}, 0.56 \%$ of $\mathrm{Mn}, 0.052 \%$ of $\mathrm{Cr}, 0.013 \%$ of $\mathrm{Cu}$, and the balance of Fe, was selected as raw materials for tension 

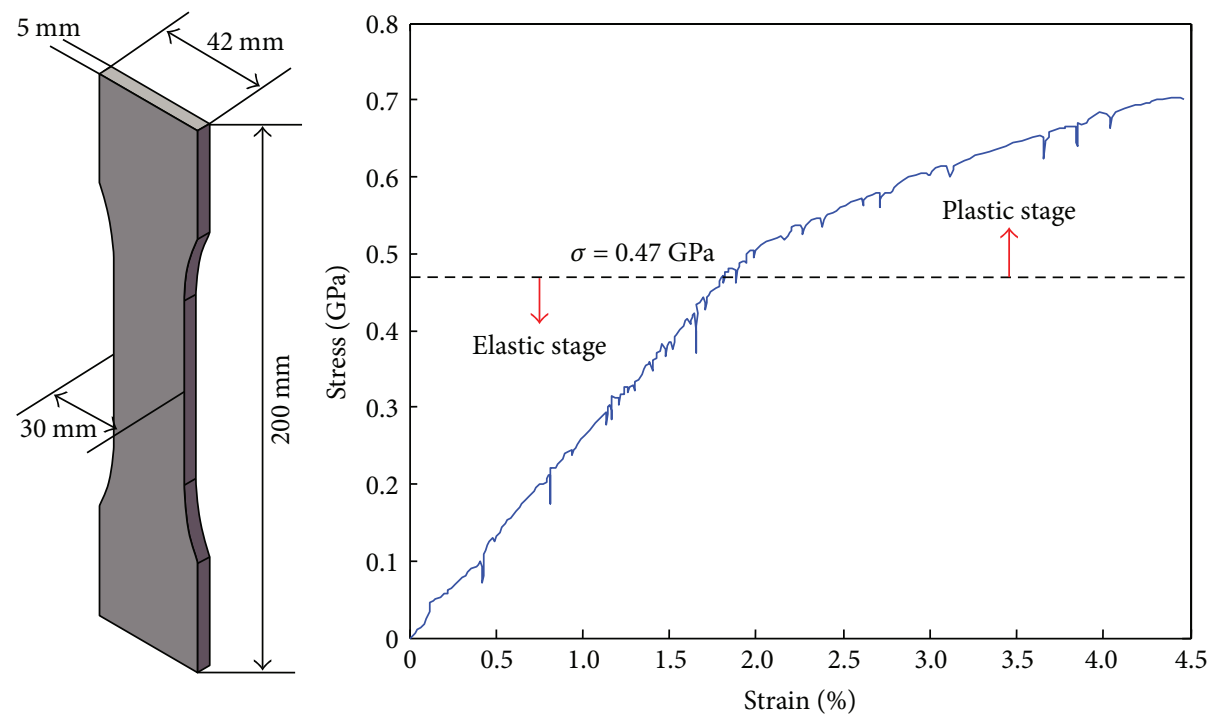

FIGURE 3: Sizes of the tensioned sample and the obtained stress-strain curve.

sample preparation. The dimensions of the sample and the experimentally obtained stress-strain curve are shown in Figure 3. The estimated yield stress is around $0.47 \mathrm{GPa}$. During the tension process, the developed PIA was used to measure the impedance of an eddy current sensor, which is mounted onto the surface of the tensioned sample. For each tensile stress level, three repeated measurements of ECS impedance were performed.

The previously reported results indicated that the inherent influencing mechanism of the variation of ECS impedance in the plastic stage was different from that in the elastic stage $[14,30]$. Thus, the analysis of the variation of ECS impedance was divided into two stages. The measured impedance curves corresponding to the stress of $0 \mathrm{GPa}$ and $0.47 \mathrm{GPa}$ were selected as the baseline for calculating the variation of ECS impedance at the elastic and plastic stages, respectively. The calculated variations of averaged amplitude curve for various stress levels are shown in Figure 4.

When the operational frequency is higher than $105 \mathrm{kHz}$, the eddy current sensor becomes a capacitive element from the initial inductive state due to the self-resonance of the circuit. In the elastic stage, a rising trend of the amplitude variation with the increase in the applied tensile stress can be observed in the inductive state of the ECS, whereas a declining trend is observed in the capacitive state (Figure 4(a)). The observed trend of the amplitude variation induced by the increase in tensile stress in the plastic stage is diametrically opposed to that in the elastic stage (Figure 4(b)). With the increase in the tensile stress, the amplitude variation of the ECS decreases in its inductive state and increases in its capacitive state.

When the sample is successively tensioned from the elastic stage to the plastic stage, both the increasing tensile stress and the decreasing thickness of sample can result in the changes of the measured impedance curves. Xie et al. [30] reported a numerical simulation method for predicting pulse eddy current signal, and they found that the eddy current signal changes due to only the thickness variety are much smaller than the changes caused by residual plastic strain. Hence, in the study, influence of thickness of tested sample is ignored and the obtained results in Figure 4 are used for conducting quantitative analysis of the dependency of impedance parameters on the applied tensile stress. The relationship between the variation of amplitude and the applied tensile stress in the elastic stage depends on the operating frequencies. In the frequency range of $60 \sim 100 \mathrm{kHz}$, linear fitting tool is applied to analyze the measured data and the curve fitting regression coefficient is higher than 0.9 , as shown in the upper insert in Figure 5. The slope of the fitting curves can be considered as the sensitivity of ECS impedance to the stress. Here, the measured data at the frequency of $84 \mathrm{kHz}$ together with the linear fitting curve are plotted in Figure 5 and the estimated sensitivity is around $8.7 \%$ per GPa. In the frequency range of $110 \sim 150 \mathrm{kHz}$, quadratic fitting results can better illustrate the variation of the measured data than the linear fitting results. The curve fitting regression coefficient in this frequency range is shown in the right insert in Figure 5. The result obtained at the frequency of $132 \mathrm{kHz}$ shows that at the yield stress point, the variation of impedance amplitude is around $1.3 \%$, which is only one-third of the value at the frequency of $84 \mathrm{kHz}$.

Similar analysis method is applied to analyze the experimental data obtained in the plastic stage. Figure 6 shows the results obtained at the frequencies of $84 \mathrm{kHz}$ and $132 \mathrm{kHz}$. Compared to the elastic stage case, in a wider frequency range of $20 \sim 180 \mathrm{kHz}$, the relationship between the variation of amplitude and the tensile stress can be represented by quadratic equations with fitting regression coefficient higher than 0.95 (Figure 6). For each case of the investigated frequency, the variation of amplitude has an inflection point just around the yield point of the tested steel sample. For instance, at the frequency of $132 \mathrm{kHz}$, a net decrease of $1.3 \%$ in the amplitude occurs when the tensile stress increases to $0.47 \mathrm{GPa}$ from the free state; a net increase 


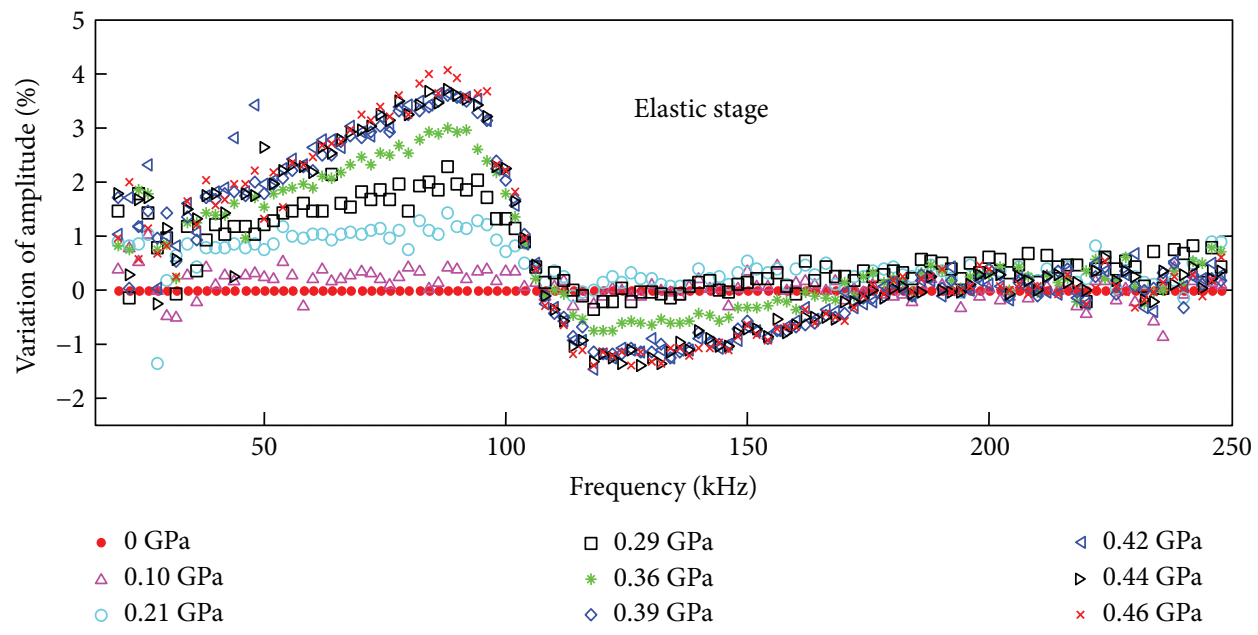

(a)

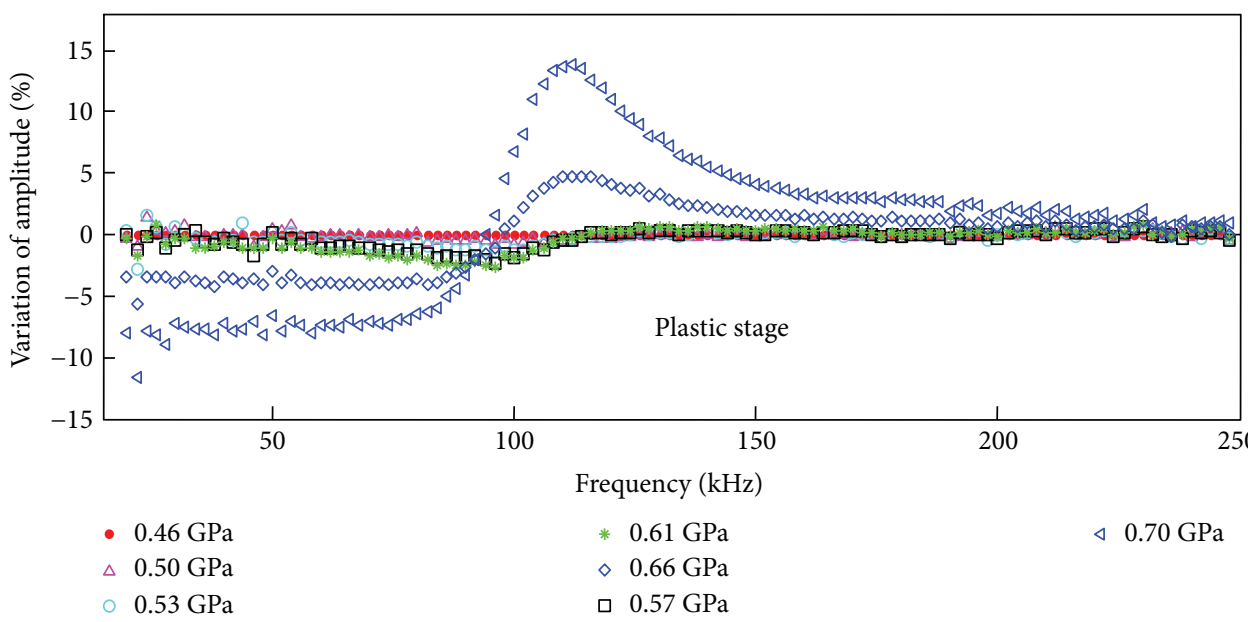

(b)

FIGURE 4: Estimated variation of averaged amplitude curve during the elastic stage (a) and the plastic stage (b) of the tensioned sample.

of $7.7 \%$ in the amplitude is achieved as the stress climbs from the yield point to around $0.7 \mathrm{GPa}$. The change in the microstructures induced by plastic deformation after the yield point significantly contributes to the variations of permeability and magnetostriction of the ferromagnetic material [25] and consequently results in greater variations of ECS impedance as compared to the case of the elastic stage. Two mechanisms, respectively, dominated by the microstructure change and tensile stress are involved in the plastic deformation stage. The above two mechanisms may have opposite effects on the variation of the magnetic properties especially the permeability, as indicated in (4) and (6).

Several other impedance parameters, which were also sensitive to the elastic and plastic deformations, were analyzed. Figure 7 shows the averaged results of phase angle variation for all the tested stress levels, and the results are categorized in the elastic and plastic stages to clearly show the opposite trends. In the entire tested frequency range, the monotonous climbing or descending trend of the impedance amplitude with the increase in the tensile stress can be inferred from the results in Figure 6. Unlike the results in Figure 6, in the frequency range higher than $130 \mathrm{kHz}$, the variation of phase angle induced by the increasing tensile stress becomes complicated, demonstrating an oscillation behavior in the plastic stage and randomly jumping behavior in the elastic stage (Figure 7). Hence, it is better to analyze the results in the frequency less than $130 \mathrm{kHz}$.

Here, the data obtained at the frequencies of $96 \mathrm{kHz}$ and $120 \mathrm{kHz}$ were selected for discussion. The measured phase angle for free sample case is used as the baseline for all the stress level cases to calculate the phase angle variation in percentage. As indicated in the insert in Figure 8, similar changing trend of the phase angle variation induced by the tensile stress can be observed at the frequencies of $96 \mathrm{kHz}$ and $120 \mathrm{kHz}$. The applied tensile stress corresponding to the inflection point of the phase angle variation at $96 \mathrm{kHz}$ is around $0.5 \mathrm{GPa}$, which is close to that at $120 \mathrm{kHz}, 0.47 \mathrm{GPa}$. The results in Figures 5, 6, and 8 apparently show that the continuous variation of impedance can reflect the elastic and plastic deformations of the material. The two deformation stages can be simply separated by the 


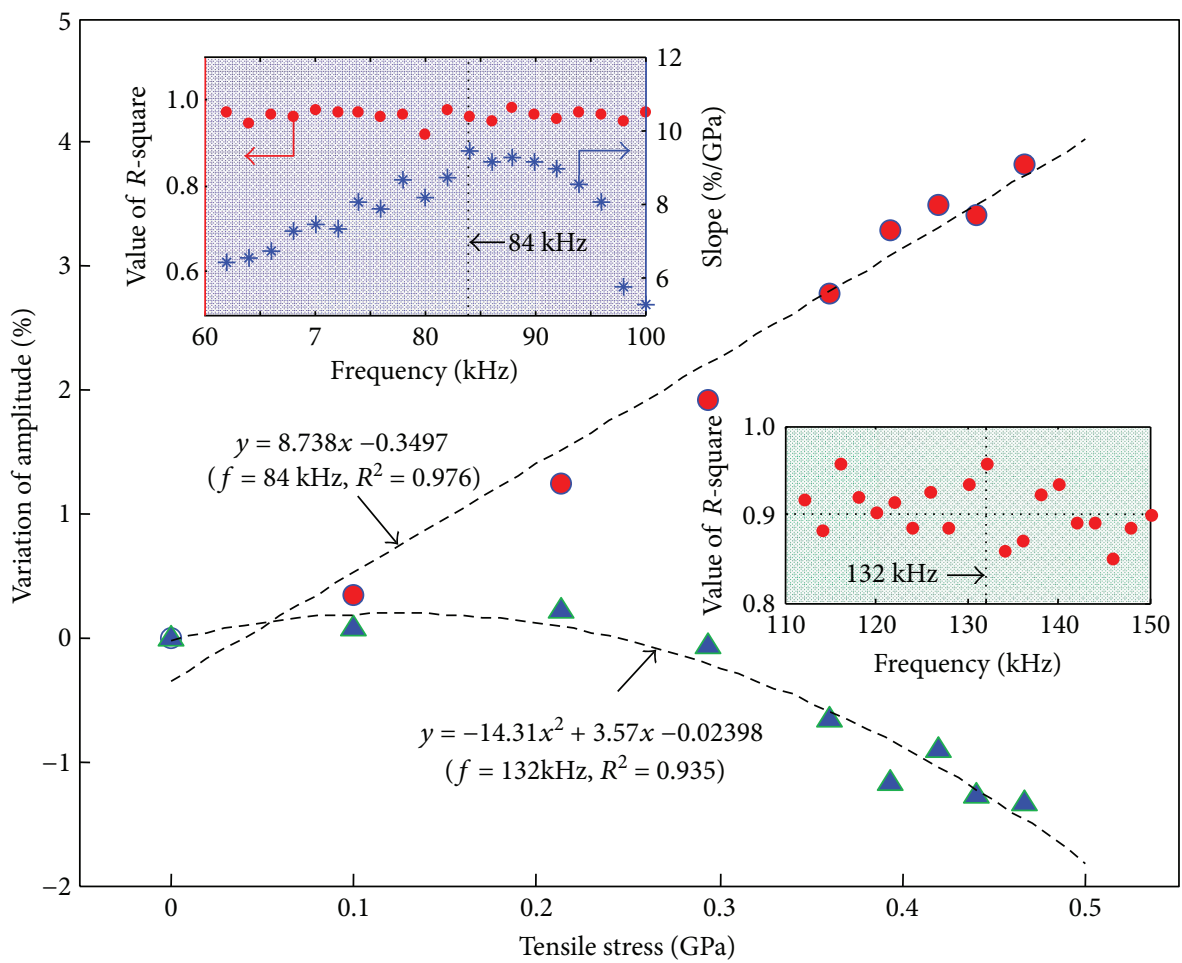

FIGURE 5: Dependence of variation of amplitude at the operating frequencies of $84 \mathrm{kHz}$ and $132 \mathrm{kHz}$ on the applied tensile stress in the elastic stage.

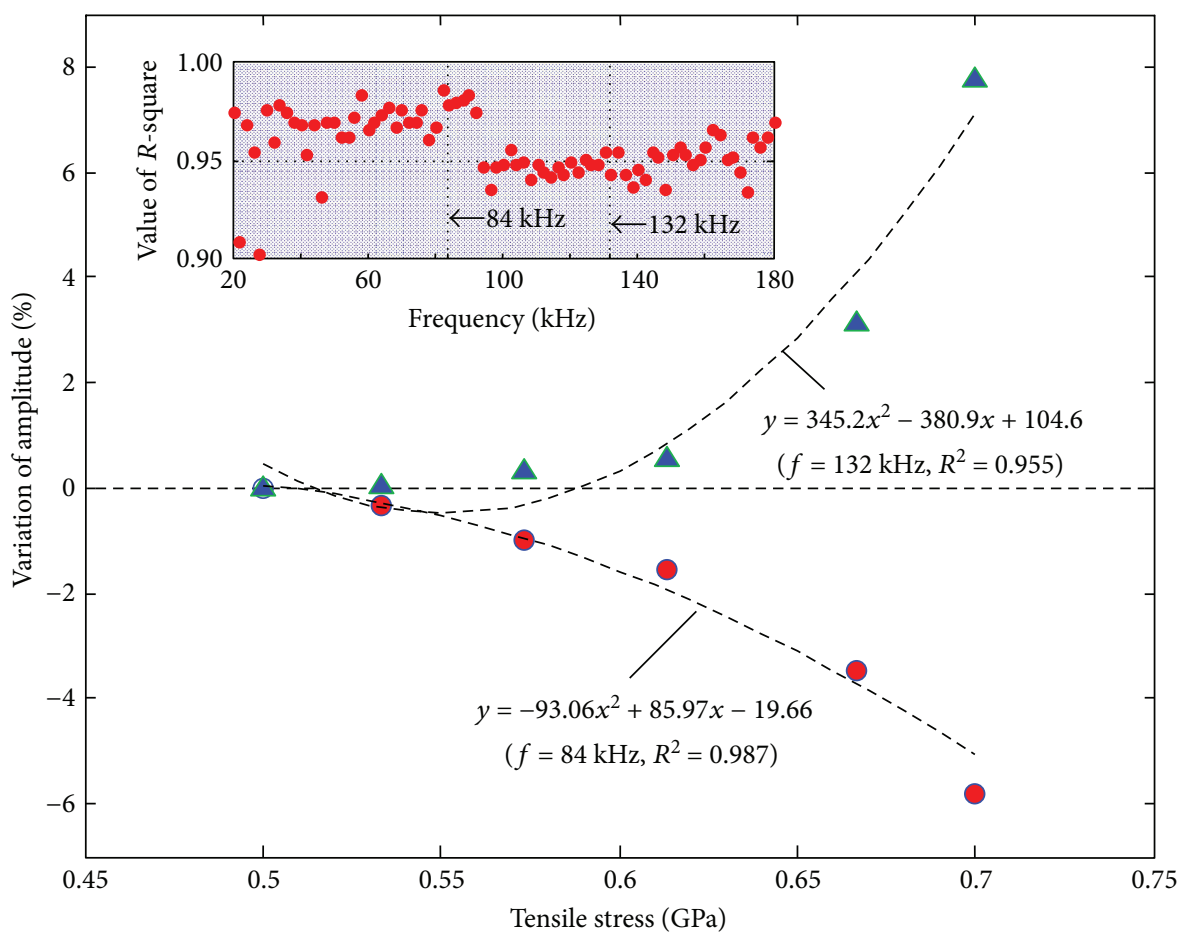

FIgURE 6: Dependence of variation of impedance at operating frequencies of $84 \mathrm{kHz}$ and $132 \mathrm{kHz}$ on the applied tension stress in the plastic stage.

inflection point of the impedance variation curves because the inflection point just corresponds to the yield stress of the material. The climbing or descending speed of curve of phase angle variation depends on the frequency. For instance, in the elastic stage, the descending speed of phase angle variation at $120 \mathrm{kHz}$ is faster than that of $96 \mathrm{kHz}$ and the opposite conclusion can be found in the plastic stage. 


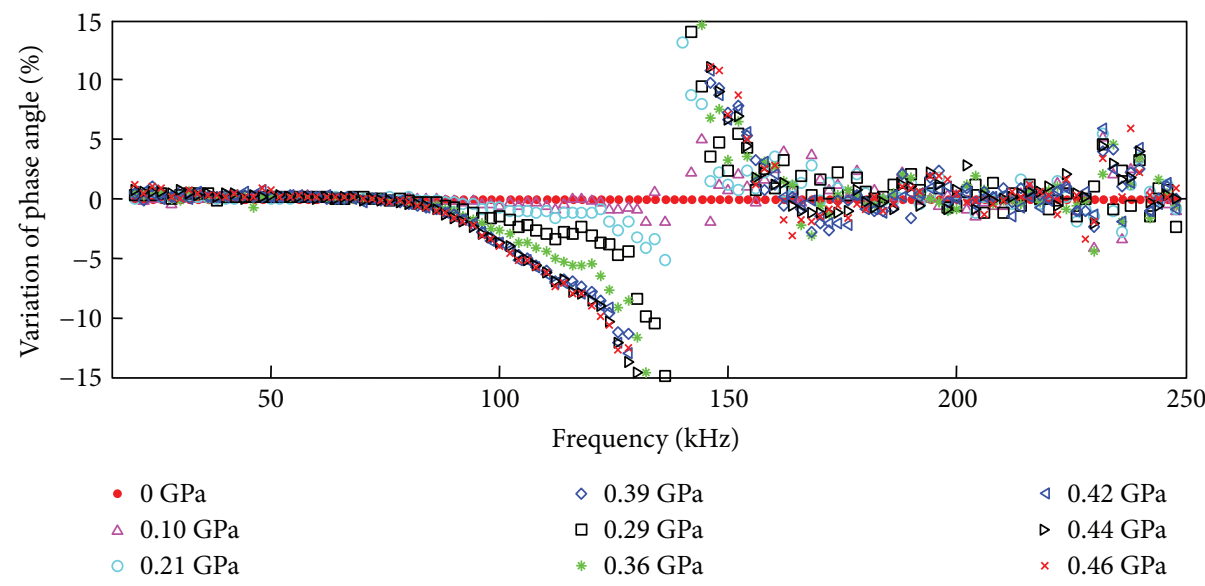

(a)

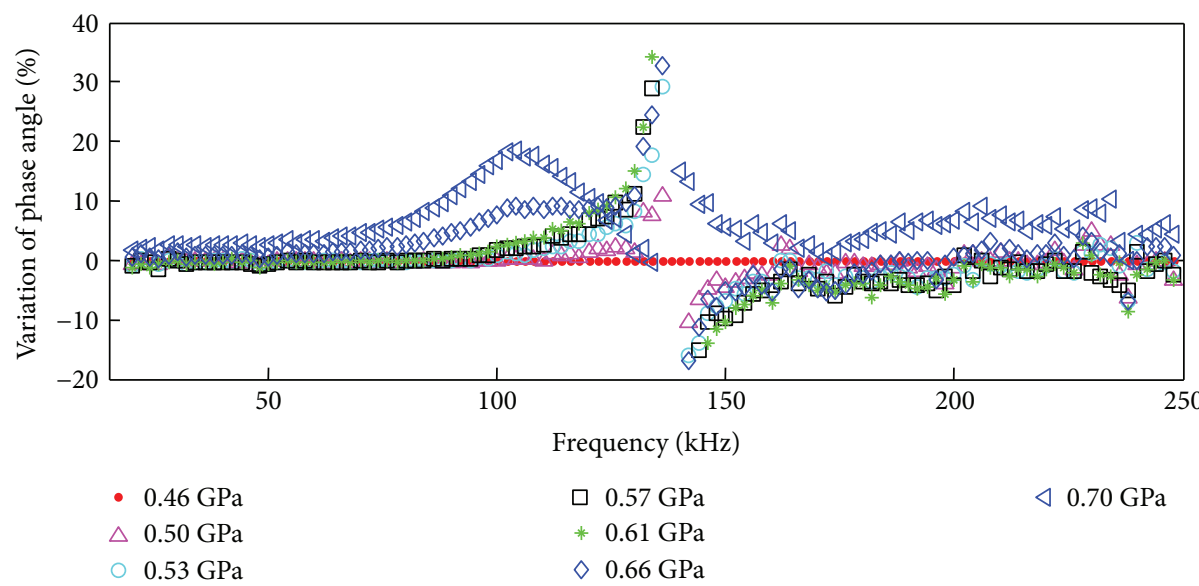

(b)

FIGURE 7: Estimated variation of phase angle during the elastic stage (a) and the plastic stage (b) of the tensioned sample.

The shift of the entire impedance curves was estimated with the variation of two featured resonate frequencies, which correspond to the peak amplitude and the phase angle of $47^{\circ}$, respectively. Curve fitting tools based on six-order Gaussian equations were applied to analyze the measured impedance data (Figure 9(a)), and then the interpolation method was applied in the measurement frequency range to refine the frequency-sweeping increment. Figure 9(b) shows the shift of the two featured frequencies when the tensile stress continuously increases from $0 \mathrm{GPa}$ to $0.7 \mathrm{GPa}$. Again, an inflection point occurs in the curves when the applied stress reaches the yield point of $0.47 \mathrm{GPa}$. The trend is consistent with the results in Figure 8. However, the shift of the two featured frequencies caused by the tensile stress of $0.1 \mathrm{GPa}$ is only hundreds of hertz. If these two featured frequencies are used as sensitive parameters for the characterization of the tensile stress, it is suggested that the incremental frequency during the impedance curve measurement be less than $100 \mathrm{~Hz}$ at the cost of the substantial increase in measurement time.

The curves in Figures 8 and 9(b) have a similar shape throughout the range of the applied stress. The variations of the amplitude at the frequency of $84 \mathrm{kHz}$ (Figures 5 and 6) were selected to help analyze the effects of tensile stress on the inductance of the eddy current loop. It is impossible to measure the exact value of the parameters of $I_{\mathrm{m}}$ and $k$, so only the stress-induced inductance variation is concerned in the study. As indicated in (2), the resistance $R_{\mathrm{e}}$ is assumed to linearly decrease with the increase in the tensile stress and the inductance $L_{\mathrm{e}}$ is proportional to the total equivalent relative permeability. With the increase in the tensile stress, the relative permeability is adjusted to ensure that the variations of real $\left(R_{\mathrm{cn}}\right)$ and imaginary $\left(X_{\mathrm{cn}}\right)$ parts of the impedance predicted with (1) are consistent with the measured ones. The results in Figures 8 and 9(b) indicate that the data scatter of the repeated measurements is quite small. Hence, only the averaged impedance values of three-time measurements are used as the references in the estimation of $R_{\mathrm{cn}}$ and $X_{\mathrm{cn}}$, and the estimated inductance $L_{\mathrm{e}}$ in percentage is shown in Figure 10.

The applied stress changes the macromagnetic behavior of the materials through changing the sizes, orientations of the domains, and the number of pinning sites. In the elastic stage, the increasing tensile stress will enhance the sample magnetization through progressively eliminating the domains, where magnetization vectors were perpendicular 


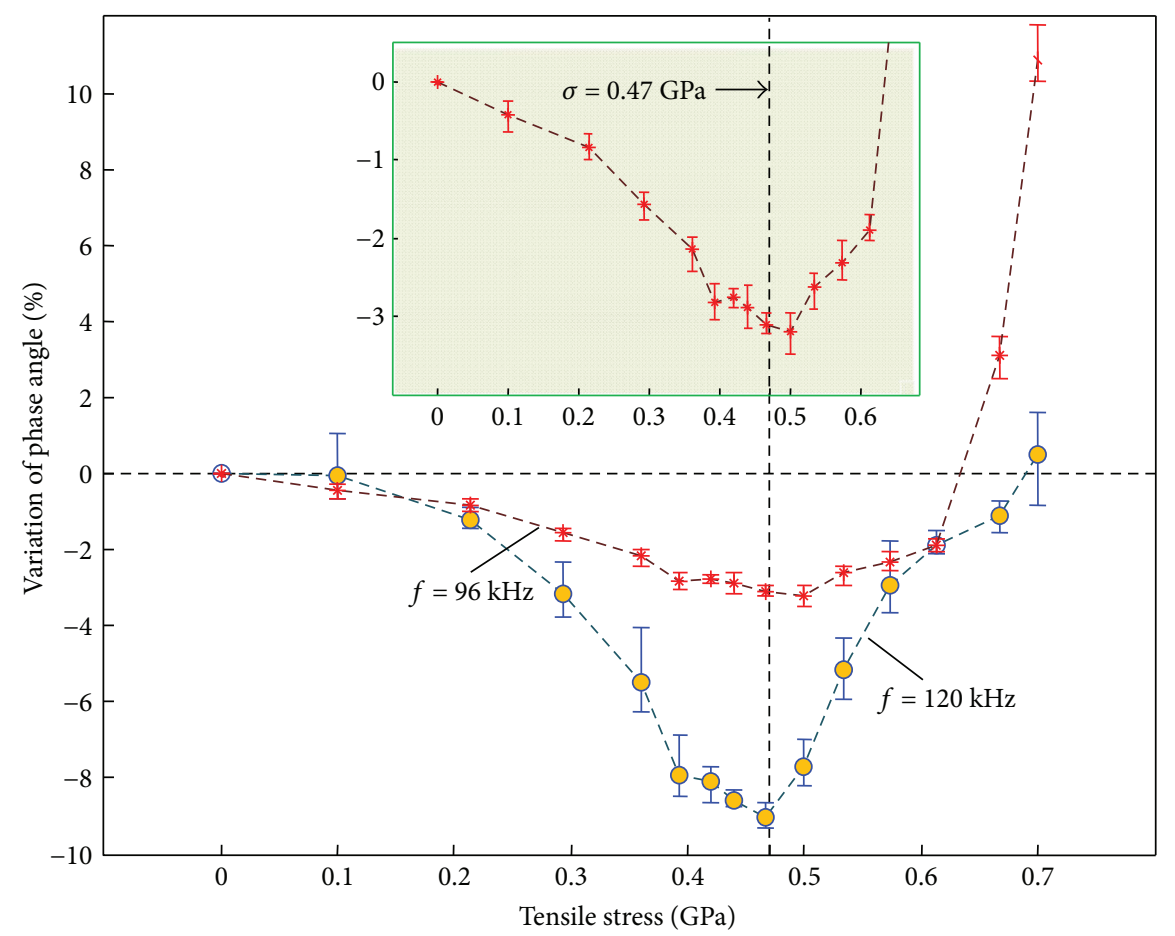

FIGURE 8: Variation of the measured phase angle as the tensile stress applied to the steel sample increases.

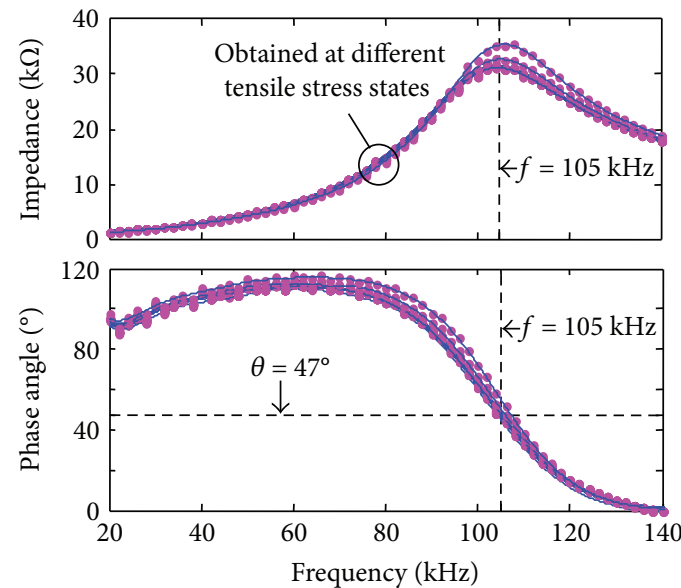

(a)

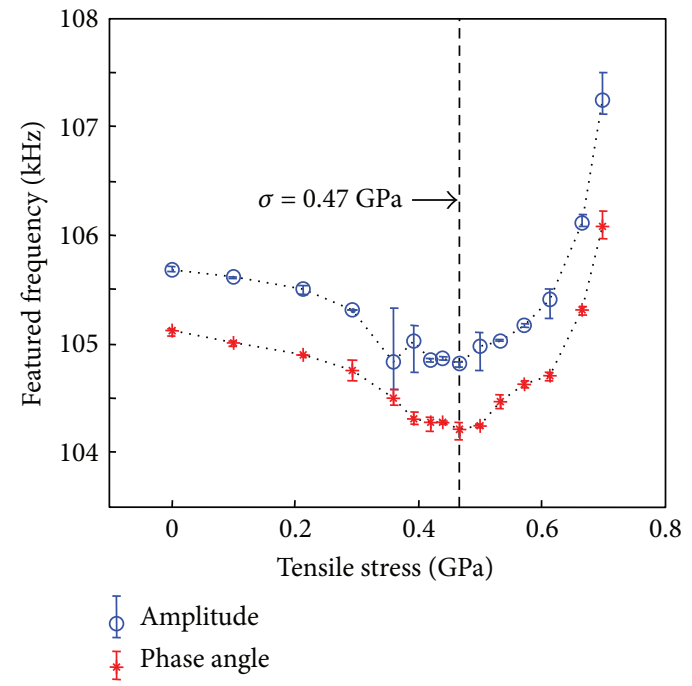

(b)

Figure 9: (a) Measured impedance curves under different tensile stress and (b) the shift of the two featured frequencies.

to the applied magnetic field [25]. As a result, the permeability of the sample is increased by reducing the total area of domain walls and the amount of domain wall pinning. Therefore, the inductance $L_{\mathrm{e}}$ tends to increase before the stress reaches the yield stress (Figure 10). After the stress overcomes the yield point, the number of pinning sites substantially increases with the increase in the dislocation density, thus weakening the strength of the magnetic field, as indicated in (6). Compared to the stress in the elastic stage, the stress in the plastic stage has a fourth-power effect on decreasing the magnetic field. Therefore, the permeability and the value of the inductance $L_{\mathrm{e}}$ decrease faster (Figure 10) with the increase in the plastic deformation degree.

The experimental procedure mentioned above is commonly used to calibrate the relationship between the impedance parameters and the applied stress. The obtained precalibration equations can be applied for evaluating tensile stress level based on eddy current techniques. However, the nonmonotonous dependency of the featured parameters 


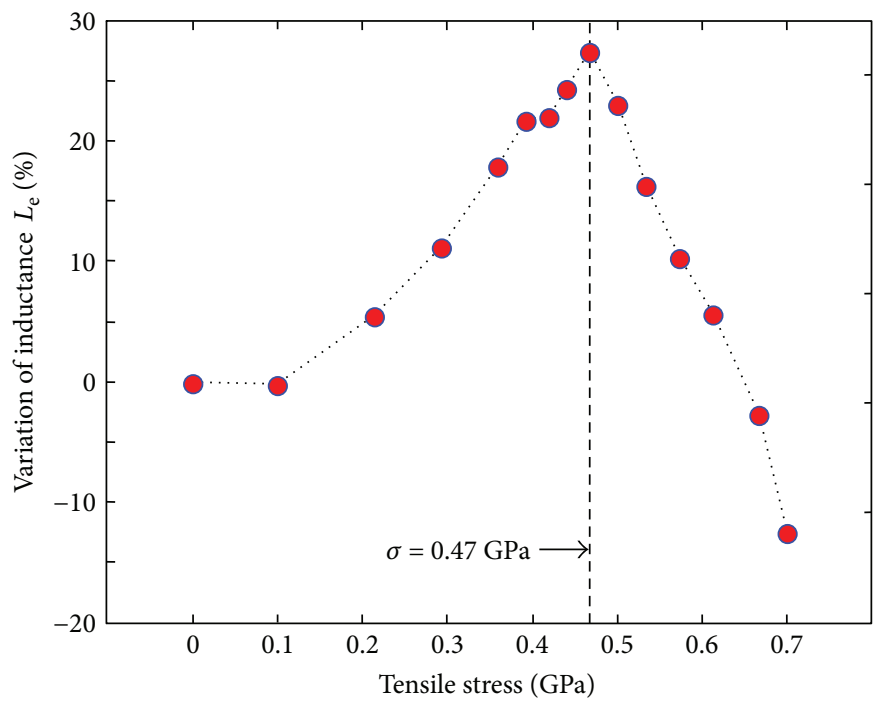

FIGURE 10: Estimated variation of inductance of eddy current loop based on measured impedance data of $84 \mathrm{kHz}$ and (1).

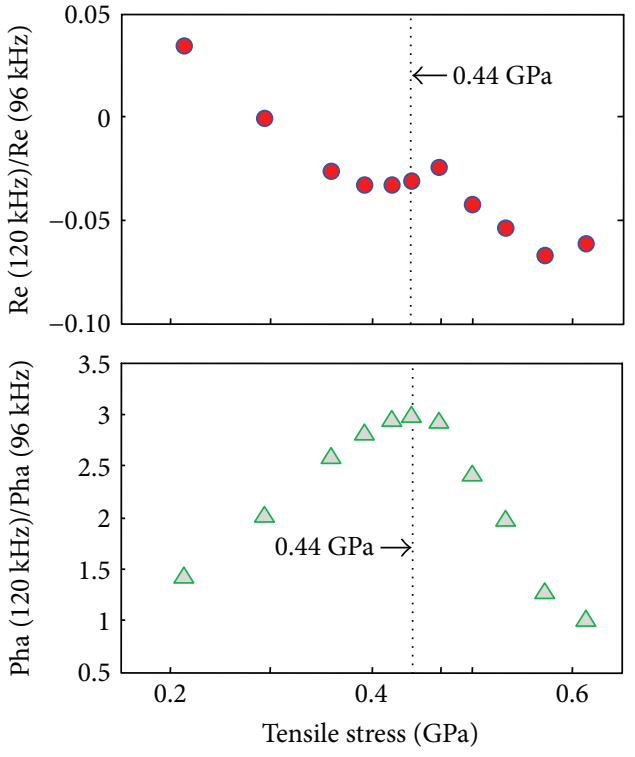

(a)

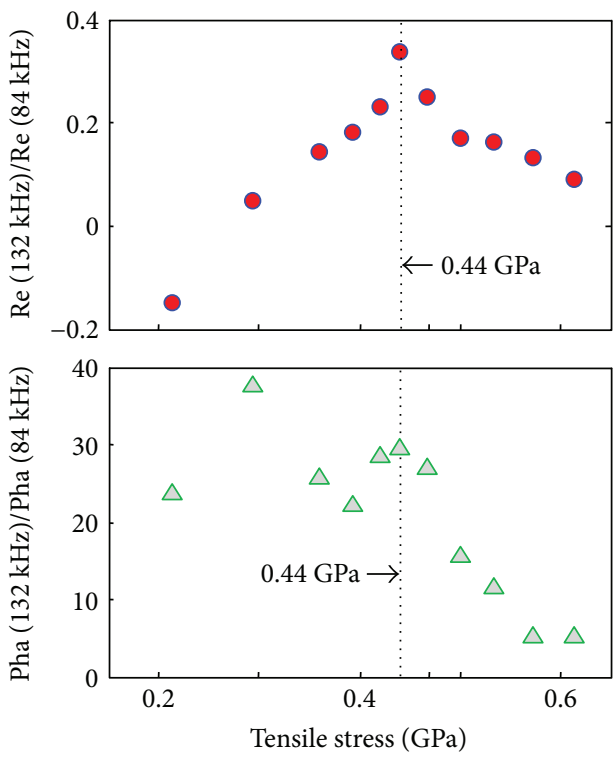

(b)

FIGURE 11: Effects of applied tensile stress on the combined parameters which are estimated from the results of frequency pairs of $120 \mathrm{kHz}$ and $96 \mathrm{kHz}$ (a) and $132 \mathrm{kHz}$ and $84 \mathrm{kHz}$ (b).

(such as resonant frequencies, amplitude, or phase angle variation) on the stress shown in Figures 6, 8, and 9 cannot be directly applied. Therefore, new featured impedance parameters combining the information of two frequencies were first introduced to characterize the stress variation during the entire elastic and plastic stages.

The two frequencies are individually selected from the frequency ranges which, respectively, correspond to the inductive and capacitive state of the ECS. Here, the two frequency pairs are selected as follows: $96 \mathrm{kHz}$ and $120 \mathrm{kHz}$; $84 \mathrm{kHz}$ and $132 \mathrm{kHz}$. Based on the average results of threetime measurements, the real part and the phase angle of the impedance at each frequency pair are extracted. The ratios of the extracted real parts (or the phase angles) at each frequency pair show different variations (Figure 11). For the frequency pair of $96 \mathrm{kHz}$ and $120 \mathrm{kHz}$, with the increase in the tensile stress, the variation of the ratio of phase angles follows a parabola-like trajectory and the ratio of the real parts almost monotonously decreases with the increase in the tensile stress.

Under the tensile stress of $0.44 \mathrm{GPa}$, the ratio of the real parts slightly deviates from its descending trend due to the yielding process of the material. For the frequency pair of $84 \mathrm{kHz}$ and $132 \mathrm{kHz}$, the variation of the ratio of real parts follows a hill-like trajectory, whilst the ratio of phase angles mildly trembles to descend with the increase in the tensile stress. For the carefully selected frequency pair, one of the two combined parameters demonstrates 
roughly monotonously dependency on the tensile stress during the entire elastic and plastic stages. This may provide a new solution to overcome the difficulty in identifying the elastic or plastic deformation stage of the material with the eddy current method.

However, there are prerequisites for applying the abovementioned new featured impedance parameters for practical applications due to the nature of calibration experimentbased method. More precisely, the material, rolling direction even the surface roughness of the tested sample, should be same as that of the tensioned sample in the calibration experiments. More calibration experiments will be performed in different materials in the future to explore the applicable range of the proposed new parameters.

\section{Conclusions}

The effects of applied tensile stress in both elastic and plastic deformation stages on the shift of the eddy current sensor's impedance were experimentally investigated with the developed portable impedance analyzer. The portable impedance analyzer was developed based on a TiePie-HS3 device and a connected laptop equipped with LabVIEW software. In the frequency range of $0 \sim 250 \mathrm{kHz}$, the portable impedance analyzer could provide comparable impedance measurement accuracy of the Agilent 4294a impedance analyzer.

The changing tendency of the impedance induced by the tensile stress depends on the frequency. For instance, at the frequency of $84 \mathrm{kHz}$ (inductive state), the ECS impedance amplitude variation increased with the increase in the tensile stress and finally decreases until the tensile stress increases to the yield point. An opposite variation behavior can be observed at the frequency of $132 \mathrm{kHz}$ (the capacitive state). In the entire tension process, the phase angle variation and the resonant frequencies of the ECS are sensitive to the applied stress. The featured parameters initially experienced a slow decreasing trend before the stress reached the yield point, and then they increased with the increase in the tensile stress faster than that of the elastic stage.

The estimated variations of inductance (and permeability) based on measured impedance data of $84 \mathrm{kHz}$ and an equivalent field model could be used to explain such phenomenon. The permeability of the sample firstly increased with the increase in the applied elastic stress and then decreased after the yield point. The trend might be interpreted as follows. The applied tensile stress decreased the total area of domain walls as well as the amount of domain wall pinning. The increasing tensile stress in the plastic stage led to the increasing number of pinning sites, so the permeability of the sample decreased faster with the increase in the tensile stress in this stage. The tensile stress has a fourth-power effect on the change of the strength of the magnetic field. In order to identify the elastic or plastic stage of the material according to measured impedance variations, the ratio of the real part or the phase angle of the impedance measured at two carefully selected frequencies was introduced as new parameter, which may demonstrate monotonous dependency on the tensile stress during the entire elastic and plastic loading process.

\section{Conflicts of Interest}

The authors declare that they have no conflicts of interest.

\section{Acknowledgments}

This work described in this paper was fully supported by a grant from the National Natural Science Foundation of China (Project nos. 11402008 and 11527801), Beijing Natural Science Foundation (Project no. 3154030), and China Postdoctoral Science Foundation (Project no. 2014M560029).

\section{References}

[1] P. Li, L. Cheng, Y. He et al., "Sensitivity boost of rosette eddy current array sensor for quantitative monitoring crack," Sensors and Actuators A: Physical, vol. 246, pp. 129-139, 2016.

[2] H. G. Ramos and A. L. Ribeiro, "Image pos-processing and inversion for eddy current crack detection problems," in 2016 IEEE Metrology for Aerospace (MetroAeroSpace), pp. 203208, Italy, 2016.

[3] F. Manfred, Micromagnetism and the Microstructure of Ferromagnetic Solids, Cambridge University Press, Cambridge, UK, 2003.

[4] R. A. McCurrie, Ferromagnetic Materials: Structure and Properties, Academic Press, London, UK, 1994.

[5] D. Mercier, J. Lesage, X. Decoopman, and D. Chicot, "Eddy currents and hardness testing for evaluation of steel decarburizing," NDT \& E International, vol. 39, no. 8, pp. 652-660, 2006.

[6] S. Konoplyuk, "Estimation of pearlite fraction in ductile cast irons by eddy current method," NDT \& E International, vol. 43, no. 4, pp. 360-364, 2010.

[7] M. Zergoug, S. Lebaili, H. Boudjellal, and A. Benchaala, "Relation between mechanical microhardness and impedance variations in eddy current testing," NDT \& E International, vol. 37, no. 1, pp. 65-72, 2004.

[8] B. A. Abu-Nabah and P. B. Nagy, "High-frequency eddy current conductivity spectroscopy for residual stress profiling in surface-treated nickel-base superalloys," NDT \& E International, vol. 40, no. 5, pp. 405-418, 2007.

[9] R. Chandrasekar, A. M. Frishman, B. F. Larson, C. C. H. Lo, and N. Nakagawa, "Effects of microstructure on eddy current residual stress characterization of shot-peened Inconel 718," JOM, vol. 64, no. 2, pp. 257-264, 2012.

[10] G. Y. Tian, M. Morozov, and S. Takahashi, "Pulsed eddy current testing of thermally aged and cold-rolled Fe-cu alloys," IEEE Transactions on Magnetics, vol. 49, no. 1, pp. 517-523, 2013.

[11] K. Liu, Z. Zhao, and Z. Zhang, "Eddy current assessment of the cold rolled deformation behavior of AISI 321 stainless steel," Journal of Materials Engineering and Performance, vol. 21, no. 8, pp. 1772-1776, 2012.

[12] R. Hughes, Y. Fan, and S. Dixon, "Near electrical resonance signal enhancement (NERSE) in eddy-current crack detection," NDT \& E International, vol. 66, pp. 82-89, 2014.

[13] V. Giurgiutiu and B. Xu, "Development of a field-portable small-size impedance analyzer for structural health monitoring using the electromechanical impedance technique," Proceeding SPIE 5391, Smart Structures and Materials 2004: 
Sensors and Smart Structures Technologies for Civil, Mechanical, and Aerospace Systems, 2004, pp. 774-785, San Diego, CA, USA, July 2004.

[14] A. Habibalahi and M. S. Safizadeh, "Application of pulsed eddy current technique to stress and residual stress measurement," International Journal of Advanced Design and Manufacturing Technology, vol. 7, p. 67, 2014.

[15] Y. Sekine and H. Soyama, "Evaluation of equibiaxial compressive stress introduced into austenitic stainless steel using an eddy current method," Journal of Nondestructive Evaluation, vol. 31, no. 2, pp. 99-107, 2012.

[16] M. Morozov, G. Y. Tian, and P. J. Withers, "The pulsed eddy current response to applied loading of various aluminium alloys," NDT \& E International, vol. 43, no. 6, pp. 493-500, 2010.

[17] M. Morozov, G. Y. Tian, and P. J. Withers, "Elastic and plastic strain effects on eddy current response of aluminium alloys," Nondestructive Testing and Evaluation, vol. 28, no. 4, pp. 300-312, 2013.

[18] H. C. Schoenekess, W. Ricken, and W. J. Becker, "Method to determine tensile stress alterations in prestressing steel strands by means of an eddy-current technique," IEEE Sensors Journal, vol. 7, no. 8, pp. 1200-1205, 2007.

[19] A. Dahia, E. Berthelot, Y. Le-Bihan, and L. Daniel, "A model-based method for the characterisation of stress in magnetic materials using eddy current non-destructive evaluation," Journal of Physics D: Applied Physics, vol. 48, no. 19, article 195002, 2015.

[20] W. Cai, H. E. Chen, S. Xie et al., "A study on influence of plastic deformation on the global conductivity and permeability of carbon steel," International Journal of Applied Electromagnetics and Mechanics, vol. 45, pp. 371-378, 2014.

[21] M. Morozov and G. Y. Tian, Separation of Contributions of Elastic and Plastic Strain to Pulsed Eddy Current Response Using Principal Component Analysis, p. 9, 2010.

[22] J. García-Martín, J. Gómez-Gil, and E. Vázquez-Sánchez, "Non-destructive techniques based on eddy current testing," Sensors, vol. 11, no. 12, pp. 2525-2565, 2011.

[23] F. Yu and P. B. Nagy, "Dynamic piezoresistivity calibration for eddy current nondestructive residual stress measurements," Journal of Nondestructive Evaluation, vol. 24, no. 4, p. 143, 2005.

[24] D. C. Jiles, "Theory of the magnetomechanical effect," Journal of Physics D: Applied Physics, vol. 28, no. 8, pp. 1537-1546, 1995.

[25] J. M. Makar and B. K. Tanner, "The effect of plastic deformation and residual stress on the permeability and magnetostriction of steels," Journal of Magnetism and Magnetic Materials, vol. 222, no. 3, pp. 291-304, 2000.

[26] Z. D. Wang, B. Deng, and K. Yao, "Physical model of plastic deformation on magnetization in ferromagnetic materials," Journal of Applied Physics, vol. 109, no. 8, article 083928, 2011.

[27] D. M. Peairs, G. Park, and D. J. Inman, "Improving accessibility of the impedance-based structural health monitoring method," Journal of Intelligent Material Systems and Structures, vol. 15, no. 2, pp. 129-139, 2004.

[28] R. M. F. Neto, V. Steffen, D. A. Rade, C. A. Gallo, and L. V. Palomino, "A low-cost electromechanical impedance-based SHM architecture for multiplexed piezoceramic actuators," Structural Health Monitoring, vol. 10, no. 4, pp. 391-402, 2011.
[29] B. Wu, H. Y. Wang, X. C. Liu, F. L. Meng, and C. F. He, "Virtual oscilloscope technique based device for stress measurement using eddy current method," Chinese Journal of Scientific Instrument, vol. 36, pp. 2828-2834, 2015.

[30] S. Xie, Z. Chen, H. E. Chen et al., "Evaluation of plastic deformation and characterization of electromagnetic properties using pulsed eddy current testing method," International Journal of Applied Electromagnetics and Mechanics, vol. 45, pp. 755-761, 2014. 


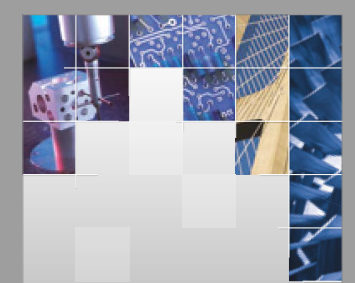

\section{Enfincering}
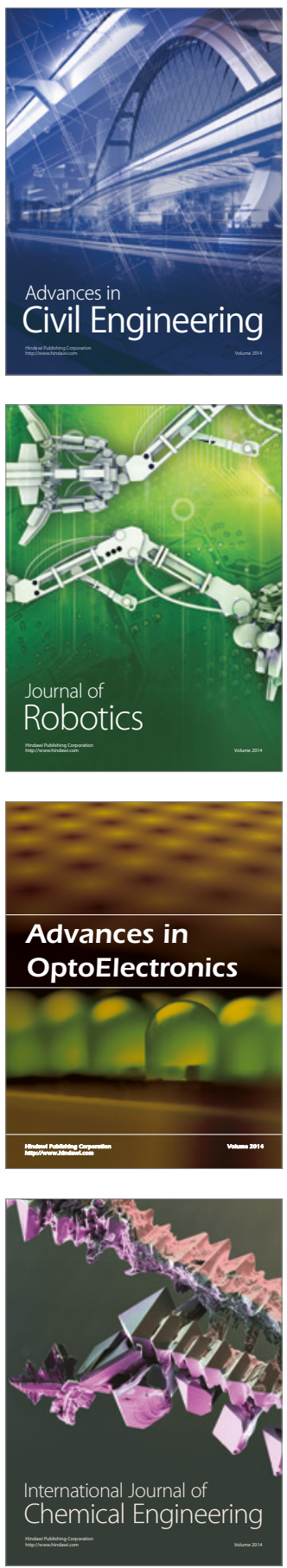

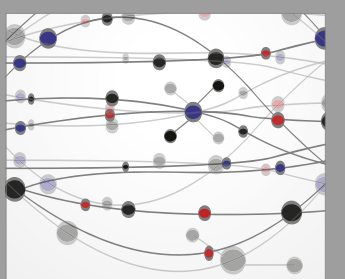

The Scientific World Journal

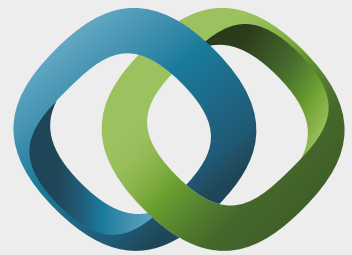

\section{Hindawi}

Submit your manuscripts at

https://www.hindawi.com
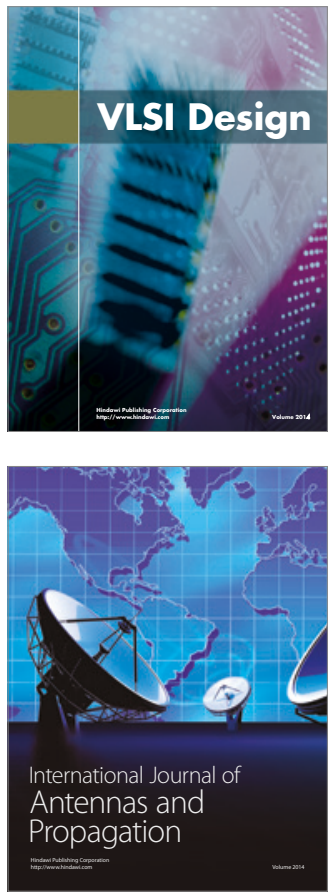

\section{Rotating}

Machinery
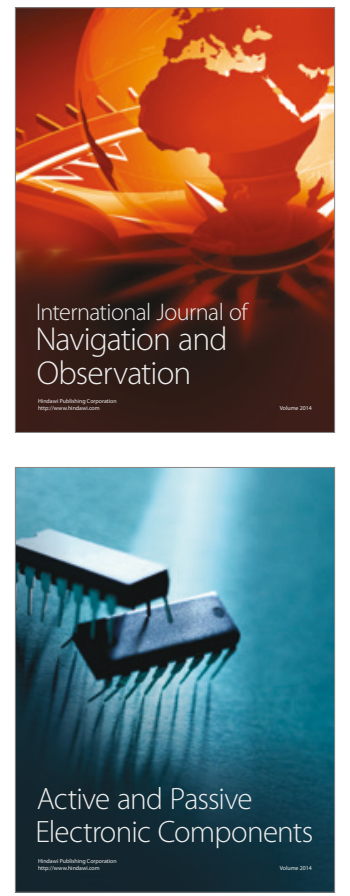
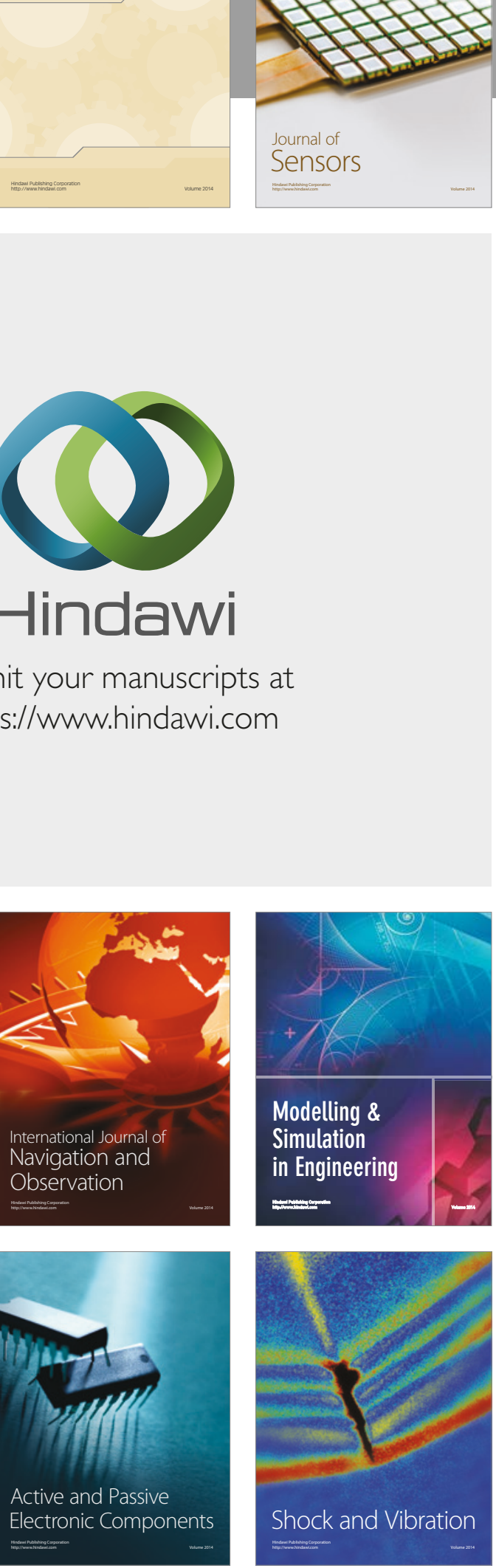
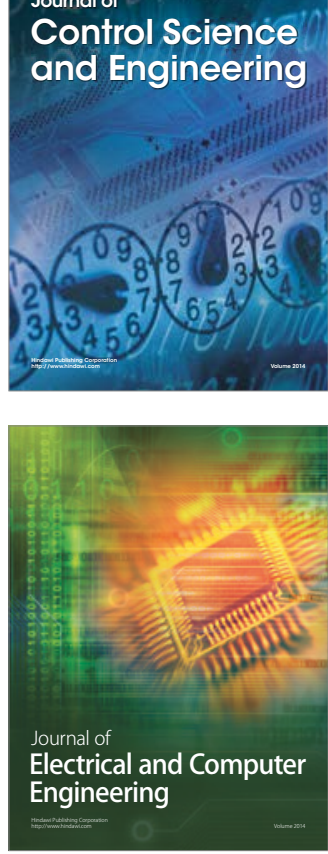

Distributed

Journal of

Control Science

and Engineering
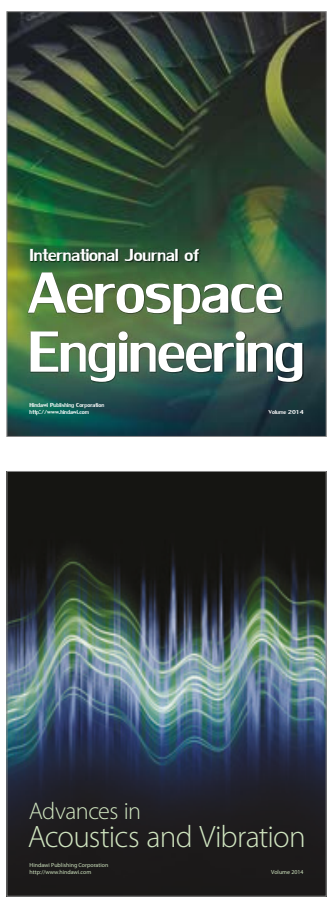

Sensor Networks 